\title{
COVID-19 infection outbreak among health care workers: Perspective from a low-middle income country
}

\author{
Nishanth Dev', Vijay Kumar², Jhuma Sankar² \\ ${ }^{1}$ Department of Medicine, Vardhman Mahavir Medical College and Safdarjung Hospital, New Delhi; ${ }^{2}$ Department of \\ Paediatrics, All India Institute of Medical Sciences, New Delhi, India
}

To the Editor

The new pandemic COVID -19 caused by Severe Acute Respiratory Syndrome Corona Virus 2 (SARS-CoV-2) is a global threat. So far, more than 11 million infections and more than five hundred thousand deaths have been reported worldwide. In India the number of cases as of $5^{\text {th }}$ July, 2020 is $6,73,165$ with 19,268 deaths.

Health care workers (HCWs) have been the backbone of this pandemic since the very beginning. However, HCWs being in the frontline are most vulnerable. In addition, they suffer from physical and mental exhaustion, agony of losing patience and colleagues, fear of infecting their family and social isolation.

Correspondence: Dr. Jhuma Sankar, Associate Professor, Department of Paediatrics, All India Institute of Medical Sciences, New Delhi, India. E-mail: jhumaji@gmail.com

Tel. +91.9818399864 .

Keywords: COVID-19; healthcare workers; SARS-CoV-2; PPE.

Contributions: All the authors made a substantive intellectual contribution. All the authors have read and approved the final version of the manuscript and agreed to be accountable for all aspects of the work in ensuring that questions related to the accuracy or integrity of any part of the work are appropriately investigated and resolved.

Funding: None.

Conflict of interest: The Authors declare no conflict of interest.

Acknowledgements: We would like to sincerely acknowledge the contributions of Miss Taniya Sharma our research staff for her contribution to this manuscript and who was one of the many health care workers affected by Covid-19 at All India Institute of Medical Sciences, Delhi. We would also like to pay our tribute to our sanitary supervisor Mr. Babu Lal from All India Institute of Medical Sciences, Delhi, a sincere, hardworking, much loved and revered person who lost the battle against COVID-19 and left a void in his place.

Received for publication: 26 June 2020.

Accepted for publication: 6 July 2020 .

${ }^{\circ}$ Copyright: the Author(s), 2020

Licensee PAGEPress, Italy

Monaldi Archives for Chest Disease 2020; 90:1474

doi: 10.4081/monaldi.2020.1474

This article is distributed under the terms of the Creative Commons Attribution Noncommercial License (by-nc 4.0) which permits any noncommercial use, distribution, and reproduction in any medium, provided the original author(s) and source are credited.
The problem of HCWs being affected by COVID-19 is a constant and ongoing concern. Currently, the total number of infected HCWs in the world is unknown and in India, it is to the tune of $\sim 25000$, although the accurate numbers are unknown. In India multiple clusters of COVID-19 among HCWs have been reported since the beginning of the pandemic.

The first cluster of infection among HCWs was reported from Rajasthan on $16^{\text {th }}$ April in a popular Indian Daily from two wellknown medical colleges in Jaipur and Bhilwara. Of 21 cases from Jaipur till 22 $2^{\text {nd }}$ April, 2020, 12 were doctors and 9 were nurses and support staff. In Bhilwara 7 doctors from a single hospital were reported to be positive. The source of infection was the HCWs. This was the first incident that prompted large scale implementation of training of HCWs in taking adequate precautions. It also prompted the hospital administrations and policy makers to procure adequate PPE for HCWs and make arrangements to tide over the acute crisis that may arise by rearranging, pooling of available health care staff for contingency.

Following these reports, similar clusters of infection were reported from several parts of the country. Notable among these clusters are a cluster from Mumbai in which more than 50 people got infected in a private hospital in Mumbai. As of now, more than $2000 \mathrm{HCWs}$ have tested positive in the capital city New Delhi. As many as 5000 doctors and 11,000 HCWs have been reported infected in the state of Maharashtra alone. As the pandemic continues, the hospitals are becoming hot spots for disease spread and poses a serious threat.

In comparison, the infection rates among HCWs in Italy and Spain are reported to be $9 \%$ and $14 \%$, respectively. As many as 151 doctors and 40 nurses have died due to COVID-19 in Italy as reported in an article by Nava et al. Maximum fatality was seen between 60-80 years of age which accounted for almost $86 \%$ of total deaths [1]. Although the exact data on mortality of HCW in India is not available, a study in India on COVID-19 in HCWs in India found the commonly effected age group to be 26-41 years (61\%). HCWs performing endotracheal intubation were at higher odds of being infected (adjusted odds ratio: 4.33 , 95\% confidence interval: 1.16-16.07). This was in in contrast to Italian data where doctors in the critical care units and pulmonology were less effected and contributed a minor percentage to the fatality. Majority of the Italian doctors who succumbed to COVID-19 were general practitioners (47\%), with dentists accounting for nearly $10 \%$. [1]. The data regarding the same is currently not available from India.

Such significant numbers amongst the HCWs has been attributed to rapid spread of SARS-CoV2 infection, unpreparedness, lack of proper training, continuous and prolonged working hours which was worsened due to death of a colleague which had to be compensated. Lack of proper hand hygiene has been identified as an important modifiable factor. Another important factor high- 
lighted in the article was overburdening of the health care system specially the critical care units involved in the management of respiratory failure, which would invariably require aerosol generating procedures to be performed. The burden on the health care system meant makeshift arrangements converting rooms with normal ventilation to function as critical care units. A few clusters have also occurred due to concealment of travel history.

It becomes imperative to put in place the plan of action to combat the infections amongst the HCWs. It is well known that respiratory procedures involve risk of aerosol generation and subsequently risk for COVID-19. Winck et al. has reviewed the modalities of Non-Invasive Ventilation (NIV)/high frequency nasal cannula in effort to generate the least amount of aerosol. They have suggested use of NIV through helmet with double limb circuit and good seal at neck-helmet interface along with use of more efficient respirators (Powered Air Purifying Respirators) for high risk aerosol generating procedures to minimize the risk [2].

An article by Ippolito et al. reviews the use of various medical masks and respirators as PPE in reducing the level of exposure to HCWs [3]. Various respirators which include medical mask, filtering facepiece respirator (FFR), elastomeric respirator, FFR with expiratory valve, powered supplied air respirator and atmospheresupplying respirator are currently available to HCWs. However, in India the common available ones include medical mask, FFR with or without valve. The availability of powered air purifying respirators in India is limited to certain select centers only.

Also, essential training on infection prevention strategies and proper use of PPE is of paramount importance. In order to know the exact incidence of infection, formulation of registry for reporting infection among HCWs could be made and the impact of measures taken can be measured over time. Once adequate protection and knowledge on infection prevention and control practices are ensured, then focus should be on other risk factors such as transmission between HCWs during case discussions, hand overs and lunch breaks. HCWs must follow the basic principles of social distancing among them as well as with the general public.

For resource limited countries like India with suboptimal health care infrastructure and shortage of HCWs, it is important to conserve this limited work force available in handling this pandemic in the best possible way. Every effort should therefore be taken to protect this limited health care force available.

\section{References}

1. Nava S, Tonelli R, Clini EM. An Italian sacrifice to the COVID-19 epidemic. Eur Respir J 2020;55:2001445.

2. Winck JC, Ambrosino N. COVID-19 pandemic and non-invasive respiratory management: Every Goliath needs a David. An evidence-based evaluation of problems. Pulmonology 2020;2 6:213-20.

3. Ippolito M, Vitale F, Accurso G, et al. Medical masks and respirators for the protection of healthcare workers from SARSCoV-2 and other viruses. Pulmonology 2020;26:204-12. 